Acta Hispanica (2018) 23: 213-223

\title{
MEDITACIONES SOBRE LA FRONTERA EN EL IMPERIO DE LA NEOMEMORIA DE HERIBERTO YÉPEZ
}

\author{
AdAlberto Mejía \\ Université Paul Valéry-Montpellier III
}

\begin{abstract}
Resumen: Heriberto Yépez (Tijuana, 1974) es una de las voces literarias más críticas en lo que va del siglo XXI en México. Parte importante de su obra literaria se concentra en el tema de la frontera entre México y Estados Unidos. Sin embargo, en El imperio de la neomemoria (2007) Yépez problematiza el significado de ese espacio fronterizo por medio de aspectos discursivos, identitarios y simbólicos. Tal ensayo, en clave biográfica -a través del personaje Charles Olson, a su vez biógrafo de Herman Melville-, analiza las formas en la que Occidente impone ciertos procesos de percepción espacial y de construcciones mnemotécnicas para rehacer la noción de cultura y de frontera. Bajo la comparación y el contraste con otras manifestaciones literarias y performances alrededor de las fronteras norte y sur de México, el objetivo de este artículo es proponer reflexiones a preguntas como: ¿De qué maneras se delimita realmente una frontera? ¿Es posible pensar por separado la frontera norte y sur de México? ¿Qué significa devenir un "ser fronterizo"? Así, como hiciera Michel Butor en À la frontière (1996) con su tipología atendiendo las causas y consecuencias, se busca reflexionar sobre la plasticidad conceptual y cultural del término frontera.
\end{abstract}

Palabras clave: Heriberto Yépez, literatura mexicana, neomemoria, tipología de frontera, fronteras norte/sur

\begin{abstract}
Heriberto Yépez (Tijuana, 1974) is one of the most critical voices in Mexican literature of the 21 st century. An important part of his literary work focuses on the border between Mexico and United States subject. However, in El imperio de la neomemoria (2007) Yépez problematizes the meaning of that border space through discursive, identity and symbolic features. Such essay, in a biographical style -via Charles Olson character, who is at the same time Herman Melville's biographer- analyzes the ways in which Occident imposes some perception processes and mnemonics constructions to remake culture and border notions. By way of comparison and contrast with other literary manifestations and performances around northern and southern Mexican borders, this article aims to propose some reflections as: In which ways can the border be really delimited? Is it possible to think separately northern and southern Mexican borders? What does it mean to become a "border being"? Thus, as Michel Butor did in À la frontière (1996) with his typology according to causes and consequences, this work seeks to give some answers to the conceptual and cultural plasticity of the term border.
\end{abstract}

Keywords: Heriberto Yépez, Mexican literature, neomemory, border typology, northern/ southern borders 
Meditaciones sobre la frontera en El imperio de la neomemoria de Heriberto Yépez

En su poemario-ensayo titulado À la frontière (1996), el escritor Michel Butor propuso algunas variables sobre lo que puede ser, representar y significar el término de "frontera". La tipología creada por Butor obedece a referencias que van desde su propia experiencia migratoria, hasta ciertos grados de convergencia entre diferentes disciplinas. Bajo la potencia de la metáfora y sus claroscuros, Butor desenvuelve un repertorio de posibilidades fronterizas según la sombra que estas proyecten o según la perspectiva desde la que se les mire o se les habite.

Esta tipología contiene casos como: la "frontera límite" -Frontière limite- que protege del exterior e incide en la percepción de los que habitan a proximidad, definiéndose respecto a ella; la "frontera íntima" -Frontière intime- que nos condiciona como dobles y paradójicos; la "frontera espesa" -Frontière épaisse-instaurada por una nación o comité internacional y que transgrede sus alrededores formando regiones intersticiales; la "frontera franqueada" -Frontière franchie- señala las expresiones que cuestionan la frontera misma, sobrevolándola para darse cuenta de la existencia del otro; la "frontera abierta" -Frontière ouverte- que, transparente con el tiempo, forma regiones de paso, de intersticios que devienen centros; o la "frontera habitable" -Frontière habitable - como una membrana vibratoria que al contacto con otras pieles territoriales se vuelve espacio de enlaces (Butor, 1996).

Frente a algunos ejemplos de la tipología de Butor se hace evidente que los registros para leer el término de frontera pueden ser sin duda plurales. Ese repertorio de posibilidades fronterizas, al ser analizado, se orienta inevitablemente hacia una serie de problemas de carácter epistemológico, mismos que se remontan al conjunto conflictos tan profusamente abordados durante las últimas dos décadas del siglo XX. Parecía que dichos fenómenos habían encontrado al fin ciertas respuestas decisivas. Por ejemplo, una de las consideraciones y conclusiones más aceptadas fue aquella que postulaba a la frontera (refiriéndose a aquella entre México y Estados Unidos) como resultado de un fenómeno híbrido tanto en su producción como en su composición. De forma simultánea, los estudios dichos multidisciplinarios o transdisciplinarios fueron el motor de análisis y crítica, el cual acentuó significativamente la presencia de un paradigma que reivindicaba lo fragmentario, la reescritura y la deconstrucción. Ello terminó por confirmar la sucesión de proyecciones emanadas del post-modernismo como la brújula para adentrarse en la frontera norte de México. 
Alfonso de Toro abordó con lucidez tal contexto, reflexionando en parte de sus estudios sobre lo producido durante ese periodo. Por ejemplo, tanto en "Hacia una teoría de la cultura de la «hibridez» como sistema científico transrelacional, «transversal» y «transmedial»" (2004) como en "Figuras de la hibridez: Carlos Fuentes, Guillermo Gómez Peña, Gloria Anzaldúa y Alberto Kurapel” (2005), de Toro analiza los giros epistémicos de dos términos fundamentales. Por una parte el de hibridez, como "un movimiento nómada de fenómenos culturales con respecto al «Otro» y a la «Otredad» $[. .$.$] un movimiento recodificador e$ innovador entre lo «local» y «lo externo»" (2004:301); por otra parte se encuentra el término "trans", prefijo que "exige una disciplina pero entrelazada en una red de parámetros [...] Se trata de un diálogo concentrado en objetos o preguntas comunes" (2004:288). De esta forma, agrega de Toro, "trans" no implica la disolución de diferencias culturales, como tampoco la nivelación de la cultura ni el consumo controlado por procesos de globalización. Por el contrario, "se entiende como un diálogo desjerarquizado, abierto y nómada que hace confluir diversas identidades y culturas en una interacción dinámica [...]" (2004:300).

Por supuesto, todo ello representó un colofón cuyo carácter transdisciplinar se brindó al ya renovado paradigma de estudios sobre las expresiones culturales a lo largo de la frontera norte de México, con especial atención a los ejes TijuanaSan Diego y, más tardíamente, al de Ciudad Juárez-El Paso. Aportaciones decisivas como Borderlands/La Frontera: The New Mestiza (1987) de Gloria Anzaldúa, Culturas hibridas. Estrategias para entrar y salir de la modernidad (1989) de Néstor García Canclini o diversos performances y publicaciones de Guillermo Gómez Peña, como Warrior for Gringostroika: Essays, Performance Texts, and Poetry (1993) o The New World Border. Prophecies, Poems \& Loqueras for the End of the Century (1996), son solo algunos ejemplos sobre la exploración del carácter, de la identidad y de la cultura fronteriza. Una importante muestra de ello se encuentra en el poema "To live in the Borderlands means you" de Gloria Anzaldúa, dentro de su libro mencionado anteriormente:

To live in the Borderlands means you / are neither hispana negra india española / ni gabacha, eres mestiza, mulata, half-breed / caught in the crossfire between the camps / while carrying all five races on your back / not knowing which side to turn to, run from [...] To survive the Bordelands / you must live $\sin$ fronteras / be a crossroads (1999:216-217). 
De esta manera, la frontera entre México y Estados Unidos se consolidaba como un enclave de encuentros identitarios, sociales, artísticos y metodológicos, produciendo sistemas transrelacionales tanto en el plano de la pertenencia (una multiplicidad o un desmarque de la pertenencia fronteriza) como en el plano de los estudios culturales. Tan es así que la noción de "Tijuana: ciudad-laboratorio" o la idea de "ciencias sociales nómadas", ambas dadas por García Canclini en su ya referido Culturas hibridas, obtuvieron rápidamente seguimiento y validez para adentrarse y comprender los fenómenos sociales, artísticos y simbólicos fronterizos de esa región específica.

La frontera norte de México se ha convertido en un foco altamente simbólico, recodificado y "transfronterizo", sobre todo gracias a las conexiones de intercambio Tijuana-San Diego y Ciudad Juárez-El Paso antes mencionadas. Las razones que han contribuido a la formación de territorios correlacionados y correlativos mantenidos por la escritura de una memoria, un imaginario y una manera de hacer "transfronteriza", pueden enumerarse de la siguiente manera: la fuerte circulación económica, los profundos problemas sociales, la exploración y redefinición identitaria, la disparidad entre zonas de uno y otro país, los desplazamientos migratorios y un muro cuya simbolización puede variar según las diferentes fantasías personales.

Ahora bien, frente a ese gran laboratorio híbrido han surgido preguntas que intentan descentralizar la observación y el significado de esa región. Las posturas "antiTijuana" o "anti-frontera híbrida" han irrumpido en la escena tanto cultural como transdisciplinaria, especialmente a través de la figura del escritor mexicano Heriberto Yépez, tijuanense nacido en 1974 y quien actualmente es uno de los críticos más importantes y radicales del país. Es un prolífico escritor que ha hecho cuento, novela, poesía, crónica y ensayo, y además tiene una presencia activa en su sitio electrónico "Border Destroyer", en el cual se autodefine como "post-mexican writer", "post-national writer in general" o "escritor anarcomunista de Tijuana".

Cuestión aparte de ese carnaval de máscaras, Yépez es autor de importantes ensayos como Made in Tijuana (2005), Tijuanologias (2006), El imperio de la neomemoria (2007) o La increíble hazaña de ser mexicano (2010), los cuales abordan, junto con otra diversidad de artículos y publicaciones, el tema de la frontera y de la ciudad faro que la ilumina: Tijuana. No obstante, una de estas publicaciones es capital por haber analizado de otra manera el tema en discusión. Es en El imperio de la neomemoria donde el autor trata el hecho fronterizo pero no desde un ángulo 
geográfico, ni social ni cultural. A través del de los preceptos y los mecanismos de una "memoria occidental", Yépez analiza el proceso de reconstrucción de una "memoria fronteriza", a la que al mismo tiempo cuestiona por suponer el resultado de un Occidente en expansión.

Resulta interesante que ya desde la década de los años noventa, Guillermo Gómez Peña se refiriera a la frontera, en Warrior of Gringostroika, como un espacio de enlace y no como una orilla, como una región cuyo monoculturalismo fuera al fin arrojado hacia los márgenes (1993). Sin embargo, a pesar de que esta tentativa buscaba redefinir y defender la hibridación cultural, regional y social, tal búsqueda desembocó, por lo bueno y por lo malo, en una visión totalizante y centralista de la frontera, alejándose poco a poco de diversos entornos que precisamente la alimentaban: el entorno político-social-identitario generalizado en México y el entorno brindado por la frontera sur del país.

De ese modo, Yépez critica la construcción de una pretendida heterotopía fronteriza (núcleo de la cultura híbrida) y de su posterior transformación en un discurso más producido por Occidente. El espacio de enlace se convertía en un espacio hegemónico que excluía otras fuentes sociales, culturales y geográficas de México y de América Latina. Cabe preguntarse, cacaso Yépez insinúa que la homogeneización y conciliación de las diferencias culturales de la frontera norte es parte de un proceso totalizante, una especie de reinicio de la memoria? El autor pone en entredicho la construcción simbólica de "Tijuana/Frontera" a la luz del Logos Occidental, cuestionando en tal símbolo algunas categorías como Origen, Memoria e Identidad. Sin duda, esto supondría un giro en los paradigmas culturales y transdiclipinarios de la frontera y su constitución simbólica, y es justo lo que busca desentrañar dentro del ensayo en cuestión.

El imperio de la neomemoria es un ensayo elaborado en diferentes registros que, relacionados entre sí, hilvanan los puntos centrales de la crítica ya comentada. En principio, el texto cuenta con cuatro divisiones muy claras. La primera de ellas, "Umbral", advierte algunos conceptos que serán desarrollados a lo largo de la exposición: términos como "cibermnémica", "Oxidente" alterando la forma correcta de escritura por una " $\mathrm{x}$ " (con evidente referencia a aquello que está oxidado, corroído), "neomemoria" y "pantopía". En este apartado se perfila una de las tesis principales: "La cibermnémica es un método de producción de imágenes sueltas. Un procedimiento de fragmentación de la realidad, la experiencia 
y la memoria [...] mediante la ilusión de la cibermnémica [...] Oxidente acumula" (2007:7).

En seguida viene el primer capítulo, "América / Pseudo-Patriarcado / Pantopía", el cual ofrece una biografía del escritor Charles Olson, cartero, escritor y eventual biógrafo de Herman Melville. La pregunta que abre este segmento es: “¿Puede una biografía explicar un imperio? Si hay una metafísica compartida, posiblemente. En la obranza de un hombre, encontrar la obranza de un imperio... Dejaré abrir la relación entre vida e imperio. Tocaré las fibras de una corpografía" (2007: 11). Para Yépez, la figura de Olson será una pieza fundamental para describir los intereses y padecimientos de la civilización norteamericana.

Tras un análisis psicoanalítico de Olson, Yépez pretende encontrar el eslabón del discurso imperialista o de la "pantopía" de los Estados Unidos, hijo ilustrado y bien educado del imperio "Oxidental". Así, Yépez transfiere los secretos psicoanalíticos de Olson a la conducción interior de los Estados Unidos, esto es: la idealización contradictoria de su padre, el miedo al cuerpo femenino (herencia de su madre) y por ende la búsqueda de un cuerpo de reemplazo, un "co-cuerpo de fantasía". Pero el riesgo de una extrapolación es latente y Yépez se da cuenta de ello al desmentir, en cierto modo, la analogía entre la vida de Olson y Estados Unidos/Oxidente que antes estableció.

Con resultados más claros, Yépez aborda el vínculo Olson-Estados Unidos con otro método. Charles Olson consagra su libro Call me Ishmael (1947) a la vida y obra de Herman Melville. Sin embargo, Olson lee la obra de Melville con el interés de quien quiere encontrar y divulgar los verdaderos valores de los Estados Unidos. Lo dicen las primeras líneas del libro: "I take SPACE to be the central fact to man born in America [...] It is geography at bottom, a hell of wide land from the beginning. That made the first American story (Park-man's): exploration" (1947:11). Enseguida aparece un fragmento más que ejemplifica la celebración de Olson al contexto ascendente de Estados Unidos durante la mitad del siglo XX: "Americans still fancy themselves such democrats. But their triumphs are of the machine. It is the only master of space the average person ever knows, oxwheel to piston, muscle to jet. It gives trajectory" (1947:12).

Es así como Yépez presenta un perfil más crítico que daría continuidad a sus tesis centrales, como aquella que enlaza el fin del primer capítulo con el segundo llamado "Co-Oxidente / Tiempo-Kinh / Imperio". En este segundo segmento, 
el autor se enfoca en la presencia de la pantopía. Es cierto que uno de los mecanismos naturales en la formación de cualquier imperio es el de abolir el pasado para reescribir la memoria; es decir, una memoria que altera la linealidad del tiempo y que privilegia el espacio de forma total y absoluta. Esta pantopía de la civilización norteamericana es señalada por Yépez cuando este se refiere al espacio "Oxidental" como aquel definido por una "acumulación topológica", un espacio que colecciona territorios, diferencias, identidades y culturas para hacerlas suyas y depositarlas en esa nueva memoria reescrita.

En el segundo capítulo Yépez realiza un ejercicio de paralelismos y contrastes. Desde una perspectiva "cuerpográfica" concibe el cuerpo de "Oxidente" como una frontera en continuo desplazamiento y como una manera de ver. El autor se aventura a establecer la presencia de diversos co-cuerpos o cuerpos complementarios de "Oxidente" formados a través del tiempo y del espacio. Así, es posible sumar a los "Oxidentes" de Europa y Estados Unidos, los diversos "Oxidentes" de México, América Latina y Oriente. De esta manera se instaura la visión marcada por una frontera movediza y que trasciende regiones, consumiéndolas: la visión de "Oxidente" o el mundo total es aquella que concibe la unidad bajo la creencia de que no existen simulaciones, ni divisiones ni diferencias conjeturales, y por ende la posibilidad de reescribir una memoria una y otra vez, de reiniciarla y hacerla nueva a partir de nuestro presente para así abordar la realidad.

Dos ejemplos precisan lo anterior. Se trata de las observaciones hechas por Antonin Artaud a los rarámuris, y de la realizada por Charles Olson a los mayas. Ambas visiones, propositivas en su intención, no pudieron escapar de la paradoja del visitante-etnólogo al arribar a una comunidad para estudiarla. El procedimiento de ambos para "recoger las huellas del pasado prehispánico" desembocó en la descripción del Buen Salvaje y de una cosmovisión exótica.

En el tercer y último capítulo llamado "Neomemoria / Modernidad-Post / Cibermnémica", Yépez se apoya en la obra de ciencia ficción de Philip K. Dick ( $\mathrm{y}$ en sus adaptaciones al cine) para evidenciar cómo los procesos mnemotecnológicos reivindican los mecanismos del imperio "Oxidental" para borrar y editar el pasado en beneficio del futuro. Lo anterior lo sustenta el autor bajo dos importantes premisas. La primera de ella es: "El sueño americano es el sueño de una nueva memoria [...] La idea de una reescritura general de los archivos habita toda nuestra civilización..." (2007:210). La segunda dice lo siguiente: 
“América es Amemoria. La sociedad norteamericana está obsesionada con personalizar, incrementar, combinar, actualizar, reconfigurar, reemplazar, o realizar reload de la reminiscencia" (2007:210).

Lo anterior forma parte de uno los puntos más importantes del ensayo de Yépez. Durante el proceso de elaboración de una "nueva memoria", mismo que es acompañado por la acumulación de espacio, la civilización "Oxidental” forma otro tipo de frontera: no una geográfica sino una que depende de la percepción entre lo real y lo mental, entre la vida tangible y la intangible, entre lo concreto y lo virtual. Esta frontera, resultado de una pantopía, es capaz de provocar "percepciones" o simulaciones identitarias, simbólicas y culturales que nacen de "memorias nuevas" y de espacios acumulados. De tal manera que la frontera para "Oxidente" funciona como un proyecto de expansión y absorción cultural, en el que las "neomemorias" instauradas constituyen las memorias y las identidades de reemplazo.

Esta zona intermedia es la que tiende un anómalo puente, un paso a desnivel, una transición o sospecha entre los dos mundos habituales del dualismo occidental, a los cuales se añade esta zona de discomunicación, de traducción traidora, frontera disfuncional, readaptación patógena, dreamland, "desierto de lo real", waiting room, no-lugar, spooky place, isla de la fantasía, middle of nowhere, realidad virtual, outerspace o what-the.fuck (2007:210-211).

El artista Francis Alÿs realizó en 1997 un performance llamado The Loop / Pacific Rim, el cual consistía en cruzar la frontera de Tijuana a San Diego realizando el trayecto de la primera ciudad a la segunda de otra manera: no en línea sino en círculo, partiendo de Tijuana hacia Centroamérica y América del Sur, llegando a Oceanía, en seguida a Asia, hasta llegar a América del Norte para finalmente dar con su destino, la ciudad de San Diego. La postura cultural de tal periplo confronta ciertos mecanismos epistemológicos que sitúan dicha frontera como un fenómeno aislado y con una memoria exclusiva su propia situación geográfica y económica. Frente a ella se pronuncia también Heriberto Yépez, quien en la parte final de su ensayo postula lo siguiente: "El anhelo de una nueva memoria se trata de un anhelo inmigrante. (Inmigrar a un país no es tanto inmigrar a otro territorio como inmigrar a otra memoria)" (2007:212). Lo anterior pone de manifiesto los procesos transrelacionales y transdiciplinarios que han sido parte de los estudios sobre la frontera norte de México, mismos que la sitúan como un acontecimiento geográfica y socialmente híbrido, es decir, como 
un espacio en el que convergen una pluralidad de identidades (véase el caso de Anzaldúa al inicio de este trabajo) y de culturas.

La crítica establecida por Heriberto Yépez, y aún en mayor medida el performance de Francis Alÿs, defienden la necesidad de observar ese fenómeno híbrido no sólo a la luz de un "discurso Oxidental", sino sobre todo mirarlo como un espacio de cuestionamiento cuya relación con la frontera sur es imprescindible. No obstante, una de las posturas constantes en Yépez señala el hecho de dejar de ser seres fronterizos, un motivo que no queda del todo claro. ¿A qué ser fronterizo se refiere Yépez? ¿A aquél formado en dentro de la cultura de la frontera norte o a aquél construido en la frontera sur? Ambos seres fronterizos no podrían ser similares pues sus búsquedas son distintas, además de que los mecanismos culturales entre las dos fronteras son muy contrastantes. Así, Yépez no estipula herramientas de análisis y enlace para extender la mirada hacia el lado sur del país, no descentraliza su mirada del norte. Y sin embargo, el autor lanza una crítica precisa sobre la manera de entender la frontera entre México y Estados Unidos en su artículo "Lo post-transfronterizo". Tal crítica, bajo otra lectura, podría extenderse hacia la frontera sur:

\begin{abstract}
[...] ¿laboratorio o abortorio de la posmodernidad? ¿Espacio de unión, amalgama y síntesis de culturas o sitio donde se hacían más evidentes las asimetrías, desigualdades y repulsión entre culturas? ¿Fusión o fisión? [...] no hay ciudades transfronterizas pero sí comunidades e individuos que habitan, cruzan, interactúan en culturas divididas por las fronteras y estructuras heterogéneas de ambos países. [Lo transfronterizo] implica una continuidad de lógicas, un sistema compartido o una zona (económica o semiótica) continua o correlativa. Pero lo que opera entre los dos lados de la fronteras son discontinuidades, fracturas o, como yo prefiero decirlo, fallas (2012).
\end{abstract}

Dar por sentado que las problemáticas de ambas fronteras del país comparten sólo conflictos de orden social, significaría arriesgar por omisión. Desde hace algunos años hasta nuestros días, diversas expresiones artísticas han atendido críticamente lo que sucede en la frontera sur. Brevemente, por ejemplo, el caso de dos novelas publicadas en 2013, La fila india de Antonio Ortuño y Amarás a Dios sobre todas las cosas de Alejandro Hernández; el testimonio que ofrece Andrés Fábregas Puig (fundador de la Universidad de Ciencias y Artes del estado de Chiapas) en su artículo "Vivir la frontera sur" (2005) sobre uno de los puntos más relevantes en la frontera de Chiapas y Guatemala, es decir, el eje migratorio 
formado entre Ciudad Hidalgo y Tecún Umán; el documental Casa en tierra ajena (2015) de Ivannia Villalobos, basado a su vez en el estudio No más muros. Exclusión y migración forzada en Centroamérica (2015) de Carlos Sandoval García. Como lo expresa Andrés Fábregas Puig dentro de su artículo anteriormente mencionado:

\footnotetext{
La frontera sur mexicana, los pueblos y culturas que la construyen cotidianamente, más allá de los puros límites geográficos, es el encuentro de México con su vocación latinoamericana. En esos términos, la vinculación mexicana con Centroamérica y el Caribe es una tendencia claramente localizable en la vida diaria de los fronterizos, y no sólo desde el ángulo comercial, sino social y cultural. La frontera sur de México es un espacio de convergencias, en el que se comparte la historia con los pueblos centroamericanos y caribeños por eso es tan significativa la frontera sur como conciencia de nacionalidad mexicana, pero también de la indudable identificación histórica que une a México con América Latina (2005).
}

Sin embargo, los brazos intervencionistas de Estados Unidos fueron incluso más allá, deshaciendo una memoria y reescribiendo otra en los países de América del sur. Como lo constata el duro catálogo histórico que Ernesto Cardenal expone en su poema "Cantiga 24. Documento latinoamericano", para observar que la frontera de los Estados Unidos es algo más que una línea, región, laboratorio o fuente de identidades: es una presencia que absorbe hasta llegar al cono sur del continente Americano. Pero también la frontera tiene alto grado de plasticidad (como lo señaló Michel Butor al inicio de este artículo) que depende de las causas que le dan origen, de las consecuencias, de cómo se le mira y se le habita. Baste como ejemplo lo que sucede en las dos fronteras de México. Sin duda, esto podría ser sólo un corolario para decir que las fallas más graves son aquellas que en la propia identidad y memoria se han dejado de ver, paradoja de una frontera íntima.

\section{Bibliografía}

Anzaldúa, Gloria. 1999. The borderline/La frontera. The new mestiza. Second edition. U.S.A.: Aunt Lute Books.

Butor, Michel. 1996. À la frontière. Paris: Éditions de la Différence.

Fábregas Puig, Andrés. 2005. "Vivir la frontera sur de México" Bovin, Philippe. Las fronteras del istmo: Fronteras y sociedades entre el sur de México y América Central. 
México: Centro de estudios mexicanos y centroamericanos. 343-349. Fecha de consulta: 25 de octubre de 2017. Asequible en: http://books.openedition.org/ cemca/732

García Canclini, Néstor. 1989. Culturas hibridas. Estrategias para entrar y salir de la modernidad. México: Grijalbo.

Olson, Charles. 1947. Call me Ishmael. United States of America: Cornwall Press.

Toro, Alfonso de. 2005. "Figuras de la hibridez: Carlos Fuentes, Guillermo Gómez Peña, Gloria Anzaldúa y Alberto Kurapel" Mertz-Baumgartner, Birgit/Pfeiffer, Erna (eds.). Aves de paso: autores latinoamericanos entre exilio y transculturación. Frankfurt am Main: Vervuert. 83-103.

Toro, Alfonso de. 2004. "Hacia una teoría de la cultura de la hibridez como sistema científico transrelacional, transversal y transmedial" Texto Crítico 25/26. 197-235.

Yépez, Heriberto. 2012. "Lo post-transfronterizo" Literal, "Trans-border/ Trans-fronterizos", 21, abril. Fecha de consulta: 28 octubre de 2017. Asequible en: http://literalmagazine.com/lo-post-transfronterizo/

Yépez, Heriberto. 2007. El imperio de la neomemoria. México: Almadía. 